\title{
Piloting a Culturally Adapted Arabic Structured Small-Group Education Program for Adolescents with Type 1 Diabetes
}

\author{
Nehad Taha Naglaa Mesbah Zahra Rahme Dina Omar Faten Sukkar \\ Department of Education and Training, Dasman Diabetes Institute, Kuwait, Kuwait
}

\section{Highlights of the Study}

- It is necessary to study aspects of diabetes education that contribute to glycemic control.

- This study reports on the impact of a culturally adapted Arabic structured educational program for adolescents with type 1 diabetes through exploring their experiences and perspectives and those of their caregivers.

\section{Keywords}

Type 1 diabetes - Structured diabetes education .

Psychosocial impact · Adolescents · Arabic educational interventions

\section{Abstract}

Background: Kuwait has a high prevalence and incidence of type 1 diabetes (T1D). At present there is a need for structured educational programs (SEP) specifically tailored for Arabian youth with T1D. This SEP was locally and culturally adapted from the Kids In Control of Food (KICk-OFF) course delivered at the Dasman Diabetes Institute, Kuwait. Aims: To explore the psychological and social impact of a pilot SEP for Arabian youth with T1D and their mothers through exploring their respective perspectives. Methods: All 20 participants were interviewed using semistructured interviews individually, with a duration of approximately 30-45 min. The interviews were recorded and then transcribed verbatim. Data were analyzed using an inductive thematic approach. Results: The adolescents gained more self-confidence in managing their diabetes and their mothers were more confident in allowing their children take the lead in their diabe-

\begin{tabular}{ll}
\hline KARGER & $\begin{array}{l}\text { Karger } \\
\text { Published by S. Karger AG, Basel The Author(s) }\end{array}$ \\
karger@karger.com & This is an Open Access article licensed under the Creative Commons \\
www.karger.com/mpp & $\begin{array}{l}\text { Attribution-NonCommercial-4.0 International License (CC BY-NC) } \\
\text { (http://www.karger.com/Services/OpenAccessicense), applicable to } \\
\text { the online version of the article only. Usage and distribution for com- } \\
\text { mercial purposes requires written permission. }\end{array}$
\end{tabular}

tes self-management. Participants were empowered with adequate knowledge about the underlying pathophysiology of glucose metabolism and the nutritional and pharmacological management which made them feel in control and helped them to better cope with diabetes. It reduced diabetes-related family stress and gave them more social freedom, supporting behavioral change for a better lifestyle. Conclusions: Attending the adapted SEP had a positive impact on adolescents with T1D and their mothers through enhancement of their knowledge and confidence, thus giving them a sense of disease control. This pilot study has proven effective in terms of enhancement of confidence in diabetes management and psychosocial factors. Therefore, there is a need for a wider implementation. @ 2019 The Author(s)

Published by S. Karger AG, Basel

\section{Introduction}

Kuwait has had a $200 \%$ increase in the incidence of T1D over the last 2 decades [1]. Diabetes self-management education (DSME) is an essential element of diabetes management in the youth $[2,3]$. DSME is designed to 
provide people with diabetes knowledge and problemsolving skills to be able to manage their disease. The aims of DSME include improving glycemic control, changing patients' behaviors, and improving their quality of life (QoL) [2]. Therefore, several DSME programs were developed, including Kids In Control of Food (KICk-OFF), a pediatric version of the Dose Adjustment For Normal Eating Program (DAFNE) SEP, in Sheffield Hospital in the UK. It was conducted over 5 days and designed for adolescents (aged 11-16 years) with T1D and includes family members who are involved in diabetes management.

At present, there are no published qualitative studies regarding attendee experiences and the psychosocial impact of the KICk-OFF course either in the UK or in $\mathrm{Ku}$ wait, where it was adapted to Arabian Gulf culture and delivered in Arabic. Thus, it was necessary to investigate the impact of this adapted SEP. Like the KICk-OFF course, the curriculum was designed to include: knowledge about diabetes pathophysiology, hyperglycemia, hypoglycemia, carbohydrate counting, insulin dose adjustment, management of ketones and sick days, exercise protocols, school lunch packs, and traveling [4]. It was intended to enable adolescents with T1D to develop diabetes self-management (DSM) skills and behaviors and effectively implement these in their daily lives with the aim of improving their QoL and their glycemic control. It was designed to promote DSM independence among the participants [4-6]. Results from a randomized controlled trial of KICk-OFF showed no significant change in glycemic control over 2 years. However, QoL and selfefficacy in diabetes management were significantly improved [6]. The glycemic control of poorly controlled participants was improved 6 months after the course [4]. In Kuwait, initially, the KICk-OFF course was delivered in English over 5 days by a diabetes educator, a nutritionist, and a physician who were trained and evaluated by the original trainers of the Sheffield team. Although the curriculum was user friendly, feedback (delivered in English) from the first group recommended a shorter course duration, the use of Arabic, and for the grouping and content to be more culturally sensitive. The curriculum was then translated into Arabic and the schedule was adapted to 3 days for age 11-16 years to minimize absence from school and 1 day for the parents involved in the diabetes care of the child (usually the mother). The modular structure of the SEP included interactive presentations and practical sessions. While maintaining the integrity of the original content related to the diabetes management, cultural adaptations, such as segregation by gender and omission of alcohol consumption/management, were required due to nonrelevance of the local culture. Additionally, fasting during Ramadan for T1D section and introduction of carbohydrate counting of local foods in practical sessions were incorporated. Thirty adolescents with T1D and their 30 mothers attended the adapted Arabic SEP in groups of 6 .

The focus of this study was on the participants' experiences towards the SEP itself and their perceptions of its impact on their DSM with consideration to managing and living with T1D. The primary objective of this study was to explore the psychosocial impact of a pilot SEP on Arabian youth with $\mathrm{T} 1 \mathrm{D}$ and their mothers through exploring their respective perspectives. This is a qualitative evaluation of the adapted SEP in Kuwait and its goal is to subsequently assess its effectiveness for wider implementation.

\section{Methods}

\section{Participants}

In this study, adolescents aged 11-16 years with T1D who were on multiple daily injections were included. Of the 30 adolescents and 30 mothers who originally attended the adapted SEP, 10 adolescents ( 5 males and 5 females) whose diabetes duration ranged from 2 to 3.5 years, and their 10 mothers, participated in this study. Participants of the SEP were approached through telephone calls. Interviews were conducted with as many subjects as necessary to reach data saturation. Data saturation became evident when ranges of ideas were repeated and reheard throughout the 20 interviews [7]. Identification of a data saturation point helped to avoid generalization of the results due to the small sample size [8].

\section{Procedure}

Semistructured interviews (SSI) were conducted and the adolescents and their mothers were interviewed separately to confidentially enable the interviewees' experiences to be explored deeply. The same topics were explored in all of the interviews (Appendix 1). The interviews were conducted in Arabic and digitally recorded. The audio records were then transcribed verbatim and translated to English for analysis, and this was then reviewed by the coauthors for confirmation of meaning. All of the interviews were then back-translated to Arabic to validate the authenticity and accuracy of the English transcripts that were formatted for proper English grammar and spelling. Anonymity and confidentiality were maintained. The participants were referred to using numerical digits; the letter A was added to the digit for "adolescent" and letter M to refer to "mother".

\section{Data Analysis}

An inductive thematic approach was used for the analysis [9]. The authors read and reread the printed transcripts and manually highlighted relevant words, phrases, and sentences to define codes. A coding framework was developed to capture key themes, and each theme was subjected to further analysis to identify subthemes, which was confirmed by all coauthors. Translated quotes from the 
Table 1. Main theme: confidence

Subtheme: self-confidence

Sample excerpt of an adolescent's response

"... I have become more confident about diabetes management than before. I feel that I am excellent in my diabetes knowledge..."

Subtheme: family's/mother's confidence and support Sample excerpts of an adolescent's response

"... my mom and dad trust me to go out unsupervised with my friends, because they are now confident that I know how to count my carbs, take injections, and I know how to manage my diabetes myself... after the course, I have been learning a lot which has helped me, and I also help my brother, because he also has diabetes..."

\section{Subtheme: feeling of control}

Sample excerpts of an adolescent's responses

"I feel independent and normal that I can now control my diabetes... my BG readings are improved and my Alc is better... I feel more in control of my diabetes."
Sample excerpts of a mother's response

"... he became self-reliant. I have more confidence in him... he also helps me and helps his brother who has diabetes, too."

The importance of the mother's supervision

"... I can rely on him to manage on his own, but I still try to supervise him, from an invisible corner."

Sample excerpt of a mother's response

"... after the course, she has become more in control of her meals, dosages, and sports activity..."
SSI were used to demonstrate what was meant by each theme. Triangulation of multiple perspectives and experiences of both adolescents and their mothers increased the credibility and validity of the study $[10,11]$.

\section{Results}

Twenty SSI were conducted in 10 adolescents with T1D and their 10 mothers who attended the SEP. Two main themes emerged with several subthemes through thematic analysis (Fig. 1).

\section{Confidence}

Self-Confidence

Before joining the SEP, all of the participants had doubts about their treatment decisions and the accuracy of their insulin doses. They negatively identified the feeling of dependency on others to check on their management. After attending the SEP, they felt more capable of managing treatment issues, which gave them a great deal of self-confidence (Table 1).

Confidence and Support from Family or the Mother

All of the participants reported that the beneficial outcomes of the SEP were not only limited to the adolescents but also applied to their mothers. The knowledge gained in the SEP was reflected on the mothers, who became confident that their children were making proper decisions. They even felt confident to manage their siblings with diabetes. Family confidence was shown by allowing the adolescents to go out with friends and enjoy meals while

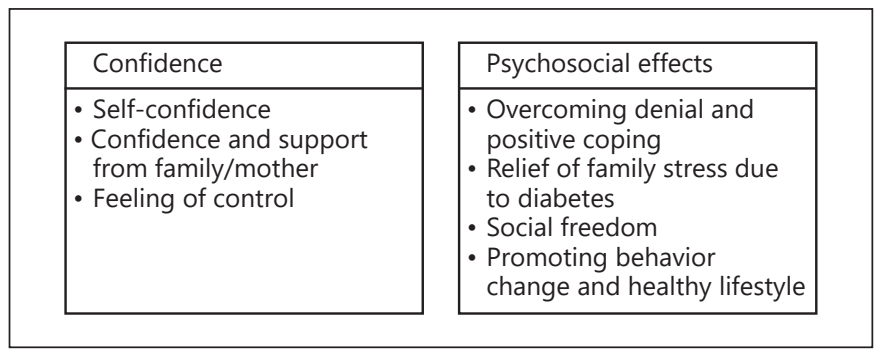

Fig. 1. Overview of the 2 main themes and corresponding subthemes.

calculating insulin doses themselves, unlike prior to the SEP wherein they felt the need for supervision. All mothers stated that family confidence does not omit the importance of mother's support and supervision.

\section{Feeling of Control}

The SEP empowered all of the participants with enough knowledge to make them feel that they were in control of their diabetes. Knowledge of the pathophysiology of diabetes was perceived as gaining control by all of the participants.

\section{Psychosocial Effect of the Program}

Overcoming Denial and Positive Coping

After being diagnosed with T1D, participants expressed their shock and feelings of shame, while being overwhelmed by all the novel information about diabetes care and management (Table 2). The feelings of emotional shock, denial, and sorrow generally led to anger and 
Table 2. Main theme: psychosocial effect of the program

\section{Subtheme: overcoming denial or positive coping}

"After the course, I felt there are a lot of young people who have the same feeling, because we were a group of young people suffering from the same condition... the course had a positive effect on me and my family."

\section{Subtheme: relief of family stress due to diabetes}

"Before the course, I used to usually argue a lot if I forgot to check my blood sugar or if my blood sugar is high... After the course, I do not argue, I just admit it if I make that mistake."

\footnotetext{
"Before the course, I was ashamed to take my injections or check my blood sugar in front of other people... However, after the course, I started to go out more confidently, and my family are more relaxed and allow me to go out on my own because of the information I gained from attending the course... Now, I have no problem dining out with my family or with my friends."
}

"He got acquainted with new friends with the same condition and became confident to inject himself in front of the people. Before the course, he just refused and felt ashamed to let anybody know about his diabetes..."
"Before the course, I barely slept... She refused to take her insulin injections. I used to check her blood sugar and give her the injection while she was sleeping. I felt so stressed and exhausted, but that changed to a great extent after she attended the course... After the course, her way of eating changed and she became aware of everything... It's not only me; all the family members were also relieved..."

"Before the course, I was afraid and worried about letting him go out with his friends to restaurants or camping or even to sleepovers at a friend's or his cousins' houses. However, now after the course, he goes out with his glucometer and his insulin and has become self-reliant... I have more confidence in him now."
Subtheme: promoting behavior change/a healthy lifestyle "Before the course, I did not use to count carbohydrates or plan my meals... After attending the course, I mostly try to choose foods with fewer carbohydrates... and now I don't need correction injections as frequently as I used to."
“... After the course, she focused on healthy food..., she got involved in sports activities, and go for walks; ... Also, she has taken control of her meals, dosages and sports activity and walks regularly..."

Sample excerpts of responses of adolescents and mothers are shown on the left and right, respectively.

isolation. Participants revealed that attending the SEP helped them to get through this phase and accept the disease.

The feeling of shame for being diagnosed with diabetes served as an obstacle, both in their management and care and on a social level, as expressed by 6 participants. Sharing stories and being with a group of people with similar ages and conditions encouraged a reduction of their sense of shame.

\section{Relief of Family Stress due to Diabetes}

Prior to the SEP, all of the participants described life with diabetes as stressful, full of arguments about injecting insulin, and blood glucose monitoring, especially in public, or even indoors. All of the participants and their mothers reported significant improvement and relief from arguments and stress over diabetes. Additionally, all of the mothers highlighted that they felt more relieved of many responsibilities of diabetes management and this was reflected in fewer family conflicts and arguments.

Experiences of Group Education for Adolescents with T1D
Social Freedom

All of the participants reported substantial improvement in their social freedom. They could participate in social gatherings and events such as dining out and sleepovers at friends' houses, family gatherings during weekends, camping, and celebrations.

Promoting Behavior Changes and a Healthy Lifestyle All of the participants explicitly stated that the knowledge they gained from attending the SEP influenced their lifestyle in several aspects including their ability to make informed choices about their meals, mainly towards healthier options, and they realized the consequences of unhealthy eating. Learning to count the carbohydrate content of meals was of immense value. This skill, which was seldom practiced, became a basic dining routine. In comparison to the original KICk-OFF, this SEP included local foods, which allowed the participants to practice this skill and master it.

Prior to the SEP, participants used to suffer from both hyperglycemia and hypoglycemia, and most of them did 
not know how to proactively adjust insulin doses in terms of physical activity both before and after exercise. After the SEP, they acknowledged feeling safer to incorporate physical activity into their lives due to their newly acquired awareness about exercise protocols. Therefore, the SEP provided the adolescents with knowledge and skills that enabled them to make proper food choices and deal with extra physical activity, which allowed them to lead and enjoy a healthy life.

\section{Discussion}

The two main themes that emerged from this explorative case study are "confidence" and "psychosocial effect," along with corresponding subthemes (Fig. 1). In terms of confidence, there was an increase in self-confidence. It has been well documented that lifestyle-based interventions result in better health outcomes [12]. Selfconfidence is a vital component of emotional well-being for adolescents. Participants expressed self-confidence as a valuable outcome of the SEP and emphasized its effect on their lives and its impact on management of their T1D. Adolescence is a peculiar stage of human development that requires special care [2]. The emotional health of adolescents is enhanced through support from healthcare teams. Enhancing the concept of "centeredness" is a way to successfully meet the emotional requirements of this stage while decreasing morbidity [2].

Additionally, there was also an increase in the families'/mothers' confidence and support. Researchers have found that education should also be directed toward mothers [13]. Both the participants and their mothers acknowledged the role of the SEP in their gains in confidence and support. The SEP enabled the mothers to have better supervision and knowledgeable involvement in treatment planning and decision making, which has been proven to be an important component of diabetes care $[14,15]$. The role of continuous parental support and involvement is crucial; however, it should not hinder the evolution of adolescents into independent persons who can take over the responsibility of their own DSM $[14,16]$. The mothers in this study were congruent with the previous idea as they reported increased levels of confidence in their children. Similarly, they highlighted that this confidence did not omit family supervision and that continuous encouragement and support are vital.

Parental support and involvement are encouraged particularly until adolescents reach maturity, and Dashiff et al. [17] discussed the importance of communication between adolescents and their parents in achieving health outcomes [16]. On the other hand, seeking to have the upper hand in their children's DSM can lead to conflicts between parents and adolescents, which in turn has negative effects on glycemic control and QoL [17]. However, Anderson et al. [15] emphasized that continuous support and involvement of parents in the DSM at the adolescence stage is just as important as in the childhood stage. This is congruent with the views of the parents in our study.

Another subtheme that emerged was the "feeling of control." The participants explicitly stated that they felt empowered and in control over their diabetes directly through the knowledge gained from the SEP. Making day-to-day DSM decisions is the most challenging aspect of having T1D, due to the complexity of diabetes management regimens [18]. The meaning of "mastering diabetes" from the patients' perspective was investigated by Ingadottir and Halldorsdottir [19], who concluded that mastering diabetes is the constant challenge of balancing medication(s) and physical and psychosocial well-being in order to live a normal daily life. Paterson and Thorne [18] demonstrated that people with T1D have to make more than 20 decisions daily to master their disease, as compared to other chronic disorders like human immunodeficiency virus or multiple sclerosis with up to 5 decisions [18]. The process of making decisions related to DSM, where blood glucose levels can be unexpected, is an experiential learning process [18].

The second theme was the "psychosocial effect of the program." Overcoming denial and positive coping were better after the SEP. King [20] denoted emotions of denial and rebellion following a diagnosis of T1D for adolescents. Denial is normally the first stage and it is challenging for both adolescents and their mothers, as demonstrated by 2 participants in this study [21]. The mothers of those participants reported that this stage reflected negatively on interfamilial relationships, adherence to medications, and DSM. Such consequences have also been demonstrated by Guthrie et al. [21]. Constraining fears of hypoglycemia and hyperglycemia, complications of diabetes are often reflected in a refusal to perform selfmonitoring of blood glucose or take insulin injections [20]. Participants revealed that attending the SEP helped them to overcome this phase and facilitated their coping with diabetes and their return to an ordinary life. Meeting people their age with the same illness and in the same situation helped them to realize that they are not alone [22]. 
Since T1D is a disease that affects the lives of patients and their families, "relief of family stress" due to diabetes was another subtheme. The family is usually impacted by demanding management regimens. The continuous care needed for the management can be a source of stress and conflict between family members. Participants stated that the SEP was helpful in relieving family stress and reduced arguments with the family and mothers over nonadherence to the DSM and/or insulin injections. The SEP was mindful of the role of the parent-adolescent relationship and its valuable effect on the DSM and has been described as being central to the healthcare of adolescents with T1D [23]. An ideal diabetes education must consider family dynamics and issues that concern the whole family and not only the affected individual [24].

While adolescents seek independence in their transition to adulthood, they experience high levels of stress from social, school, and family issues. Diabetes places an extra stress on adolescents as they are restricted from activities that are perceived as normal for their non-diabetic peers, thus affecting their "social freedom," which was a subtheme. Prior to the SEP, the participants were not allowed to enjoy camping, going out, sleepovers, and dining out with friends. The SEP empowered participants with a competency that helped their mothers to feel confidence letting them take responsibility for their DSM when they are away from direct supervision [25].

Another psychosocial effect of the program was "promoting behavior change and a healthy lifestyle." One of the essential components of mastering diabetes is dealing with desires [19]. Patients with diabetes often have inner conflicts, i.e., the desire to indulge in food temptations rather than healthy dieting habits [19]. The SEP conveyed the importance of a healthy lifestyle, physical activity, and eating habits, thus enhancing the incorporation of those habits into their daily activities. Children with T1D are more prone to suffering obesity and overweight than nondiabetic kids are. This may be due to overinsulinization, overeating, excessive intake of juices to avoid or treat hypoglycemia, and insufficient physical activity [26]. Therefore, diabetes educational courses need to consider and implement nutritional education into DSM [26].

Participants tended to adopt and incorporate positive DSM behaviors into their daily lives. The readiness to change their behavior increased. Both adolescents and their mothers expressed the importance of sharing experiences [27]. Karlsson et al. [27] suggested that participation in the KICk-OFF course commenced an empowerment process, as evident in this study. Behavioral change toward adopting a healthy lifestyle for the whole family is an important target of nutritional management of T1D. [26]. The target of behavioral change is to minimize the risk of obesity and cardiovascular complications while enhancing "psychosocial well-being" [28] and interfamilial rapport [26].

Management of T1D demands special attention and requires personal modification of different aspects of life including eating habits, physical activity, and self-monitoring of blood glucose. All children with T1D should be provided with a well-designed, age appropriate SEP, along with their parents, to prepare them to be independent and efficient in their DSM in the future. Attending the culturally adapted Arabic SEP enhanced their confidence and psychosocial status and resulted in better outcomes for our Arabian youth.

In this pilot study, empowered by knowledge, both adolescents and their mothers felt more confident in managing T1D. The SEP enhanced the feeling of control over T1D. The participants also felt empowered to cope better with T1D, thus reducing diabetes-related family stress and earning more social freedom, with a positive shift toward behavioral change for a better lifestyle.

This study demonstrated how healthcare providers and educators can empower adolescents and their mothers through this SEP. As this SEP aimed to improve the overall metabolic control and QoL, it is recommended that further studies look objectively into the metabolic control as measured by changes in $\mathrm{HbA}_{1 \mathrm{C}}$ in Kuwait. It may be of value to follow-up the cohort and collect data on their serial $\mathrm{HbA}_{1 \mathrm{C}}$ to assess the clinical effectiveness of the SEP. Given the positive findings it is recommended that this SEP be held more frequently and have a wider implementation. Moreover, other hospitals and healthcare centers should regularly host such courses.

In view of the rising incidence of $\mathrm{T} 1 \mathrm{D}$ and the increasing number of affected children and adolescents, there is the need for more trained staff. Such personnel should be able to deliver the course with consistency and efficiency.

The participants were from a single center in Kuwait, which might be a limitation to the generalization of its findings. Additionally, a small cohort of 10 patients and 10 mothers were interviewed out of the total 30 patients and 30 mothers who attended the course, which represented $33.3 \%$ of the attendees. This was considered a representative cohort; however, researchers are encouraged to replicate this study on a wider scale and/or in different settings/cultures. 


\section{Acknowledgement}

The authors thank Dr. Azza Shaltout from the Dasman Diabetes Institute for her continuous support and encouragement. The authors acknowledge the efforts of Dr. Katherine Price and Julie Knowels, the original creators of the KICk-OFF course at Sheffield Children's Hospital, UK, who also trained the team. The authors would like to acknowledge Jonathon D. Vaz for his contribution in reviewing and editing this paper.

\section{Statement of Ethics}

Informed consent was obtained from the participants and their guardians (the father of the adolescent as per the law of the State of Kuwait) were asked to sign a consent form and a minor consent form. The study protocol was approved by the Ethical Review Committee of the Dasman Diabetes Institute.

\section{Disclosure Statement}

None of the authors has any conflict of interests to disclose.

\section{Appendix 1: Interview Guide}

A while ago, you (and your child) attended a structured educational course for T1D with a group of people your age and with your condition. We are going to discuss your (and your child's) experience with this course. Our interview will be recorded. If you feel uncomfortable at any point, we can stop the interview if you wish.

Question

1. How do you feel about the control of your diabetes after attending the course?

\section{Prompt}

- Do you feel that you could manage your diabetes independently?

- What do the healthcare professionals tell you? What do your parents and family say? What do your friends say? How does that make you feel?

2. What effect did the course have on your diabetes, if any?

- $\quad$ Sick day management? Physical activity? Meals? Insulin doses? Hyperglycemia? Hypoglycemia? Variable blood sugar results?

3. In what way, if any, did attending the course affect you socially?

4. In what ways, if any, did attending the course affect you emotionally?

- $\quad$ More freedom to go out and dine out with friends alone (group activities like field trips, camping)

- Worries about your diabetes? Confidence? More or Less? Do you speak with anyone to help you manage it?

- Who supports you? (parents, friends, a diabetes educator?)

5. In what ways has being a graduate of this course impacted your family?

6. Do you now feel more in control of your diabetes? What has made you feel this way?

- $\quad$ More independence? Control? Fewer arguments?

- Family support? Friend support? Healthcare professional support? Attending the course? Can you tell me more about that?
7. In what ways, if any, has attending the course impacted your life?

- Can you tell me more about that?

- $\quad$ Managing your diabetes yourself? Is making decisions related to your diabetes yourself difficult? In what ways, if any, has it affected your lifestyle?

- In what ways, if any, do you alter your eating, exercise, sleeping habits, frequency of blood glucose monitoring?

8. How would you rate your experience with the course on a scale of 1-10 (1 being poor and 10 being excellent)?

9. Is there anything else you would like to tell me about your experience with this course? 


\section{References}

1 Shaltout, A.A., D. Wake, T.A. Thanaraj, D.M. Omar, D. Al-AbdulRazzaq, A. Channanath, et al. Incidence of type 1 diabetes has doubled in Kuwaiti children 0-14 years over the last 20 years. Pediatr Diabetes. 2017 Dec;18(8):7616.

2 Funnell MM, Brown TL, Childs BP, Haas LB, Hosey GM, Jensen B, et al. National standards for diabetes self-management education. Diabetes Care. 2012 Jan;35 Suppl 1:S101-8.

3 American Diabetes Association. Children and adolescents diabetes care: standards of medical care in diabetes. Diabetes Care. 2017; 40:S105-13.

4 Waller H, Eiser C, Knowles J, Rogers N, Wharmby S, Heller S, et al. Pilot study of a novel educational programme for 11-16 year olds with type 1 diabetes mellitus: the KICkOFF course. Arch Dis Child. 2008 Nov; 93(11):927-31.

5 Price KJ, Wales J, Eiser C, Knowles J, Heller S, Freeman J, et al. Does an intensive self-management structured education course improve outcomes for children and young people with type 1 diabetes? The Kids In Control OF Food (KICk-OFF) cluster-randomised controlled trial protocol. BMJ Open. 2013 Jan; 3(1):e002429.

6 Price KJ, Knowles JA, Fox M, Wales JK, Heller S, Eiser C, et al.; KICk-OFF Study group. Effectiveness of the Kids in Control of Food (KICk-OFF) structured education course for 11-16 year olds with Type 1 diabetes. Diabet Med. 2016 Feb;33(2):192-203.

7 Francis JJ, Johnston M, Robertson C, Glidewell L, Entwistle V, Eccles MP, et al. What is an adequate sample size? Operationalising data saturation for theory-based interview studies. Psychol Health. 2010 Dec;25(10): 1229-45.

8 Cohen L, Manion L, Morrison K. Research Methods in Education. London: Routledge Falmer; 2007. https://doi.org/10.4324/9780203029053.
9 Thomas DR. A general inductive approach for analyzing qualitative evaluation data. Am J Eval. 2006;27(2):237-46.

10 Hamilton L. Case studies in educational research [Internet]. British Educational Research Association. 2011. Available from: https://www.bera.ac.uk/publication/casestudies-in-educational-research.

11 Morse JM. Approaches to qualitative-quantitative methodological triangulation. Nurs Res. 1991 Mar-Apr;40(2):120-3.

12 Qidwai W, Ishaque S, Shah S, Rahim M. Adolescent lifestyle and behaviour: a survey from a developing country. PLoS One. 2010 Sep; 5(9):e12914.

13 Hood KK, Huestis S, Maher A, Butler D, Volkening L, Laffel LM. Depressive symptoms in children and adolescents with type 1 diabetes: association with diabetes-specific characteristics. Diabetes Care. 2006 Jun; 29(6):1389-91.

14 Laffel LM, Vangsness L, Connell A, GoebelFabbri A, Butler D, Anderson BJ. Impact of ambulatory, family-focused teamwork intervention on glycemic control in youth with type 1 diabetes. J Pediatr. 2003 Apr;142(4):409-16.

15 Anderson BJ, Brackett J, Ho J, and Laffel L: An office-based intervention to maintain parentadolescent teamwork in diabetes management: impact on parent involvement, family conflict, and subsequent glycemic control, Diabetes Care. 1999 May;22(5):713-21.

16 Wysocki T, Greco P. Social support and diabetes management in childhood and adolescence: influence of parents and friends. Curr Diab Rep. 2006 Apr;6(2):117-22.

17 Dashiff C, Hardeman T, McLain R. Parentadolescent communication and diabetes: an integrative review. J Adv Nurs. 2008 Apr; 62(2):140-62.

18 Paterson B, Thorne S. Expert decision making in relation to unanticipated blood glucose levels. Res Nurs Health. 2000 Apr;23(2):147-57.
19 Ingadottir B, Halldorsdottir S. To discipline a "dog": the essential structure of mastering diabetes. Qual Health Res. 2008 May;18(5): 606-19.

20 King KM. "It's life - but not as we know it": adolescents' perspectives of type 1 diabetes. Sunderland: University of Sunderland; 2008.

21 Guthrie DW, Bartsocas C, Jarosz-Chabot P, Konstantinova M. Psychosocial issues for children and adolescents with diabetes: overview and recommendations. Diabetes Spectr. 2003;16(1):7-12.

22 Castro D, Malivoir S, Martin D, Gagnayre R, Robert JJ. Siblings of diabetes type 1 children: impact of illness and treatment on psychological functionary of brothers and sisters - implications for therapeutic patient education. Ther Patient Educ. 2009;1(1):13-9.

23 Leonard BJ, Garwick A, Adwan JZ. Adolescents' perceptions of parental roles and involvement in diabetes management. J Pediatr Nurs. 2005 Dec;20(6):405-14.

24 Silverstein J, Klingensmith G, Copeland K, Plotnick L, Kaufman F, Laffel L, et al.; American Diabetes Association. Care of children and adolescents with type 1 diabetes: a statement of the American Diabetes Association. Diabetes Care. 2005 Jan;28(1):186-212.

25 McCallister HA. Demonstrating competence: a qualitative study of diabetes management during adolescence [dissertation]. Auston (TX): University of Texas Libraries; 2006.

26 Smart C, Aslander-van Vliet E, Waldron S. Nutritional management in children and adolescents with diabetes. Pediatr Diabetes. 2009 Sep;10 Suppl 12:100-17.

27 Karlsson A, Viklund G, Arman M, Haglund L. Teenagers' experiences of participation in an empowerment education Programme for people with type I diabetes. Scand J Caring Sci. 2007.

28 Doherty Y, Dovey-Pearce G. Understanding the developmental and psychological needs of young people with diabetes. Pract Diabetes Int. 2005;22(2):59-64. 